\title{
ON GENERALIZED HEAT POLYNOMIALS
}

\author{
C. NASIM \\ Department of Mathematics and Statistics \\ The University of Calgary \\ Calgary, Alberta T2N IN4 Canada
}

(Received March 18, 1987)

ABSTRACT. We consider the generalized heat equation of $n^{\text {th }}$ order $\frac{\partial^{2} u}{\partial r^{2}}+\frac{n-1}{r} \frac{\partial u}{\partial r}-\frac{a^{2}}{r^{2}} u$ $=\frac{\partial u}{\partial t}$. If the initial temperature is an even power function, then the heat transform with the source solution as the kernel gives the heat polynomial. We discuss various properties of the heat polynomial and its Appell transform. Also, we give series representation of the heat transform when the initial temperature is a power function.

KBY WORDS AND PHRASES. Generalized heat equation, source solution, heat transform, heat polynomial, Appell transform, generating function, Laguerre polynomial, hypergeometric function.

SUBJECT CLASSIFICATION: $\quad 35 A 05$

\section{INTRODUCTION.}

In this paper we shall establish various properties of the polynomial solutions and its Appell transforms of the generalized heat equation of the $n^{\text {th }}$ order,

$$
\frac{\partial^{2} u}{\partial r^{2}}+\frac{n-1}{r} \frac{\partial u}{\partial r}-\frac{a^{2}}{r^{2}}=\frac{\partial u}{\partial t},
$$

where $r^{2}=x_{1}^{2}+x_{2}^{2}+\cdots+x_{n}^{2}$. Also we shall give a series expansion of the generalized temperature in terms of Laguerre polynomials and confluent hypergeometric functions. Most of the results derived here are similar to the ones found in [4\&5], which are for the less general equation

$$
\frac{\partial^{2} u}{\partial x^{2}}+\frac{2 \nu}{x} \frac{\partial u}{\partial x}=\frac{\partial u}{\partial t}
$$

which in turn is a generalization of the ordinary heat equation, [7]

$$
\frac{\partial^{2} u}{\partial x^{2}}=\frac{\partial u}{\partial t} \text {. }
$$

These known results can be considered as special cases of our more general results, when $\alpha=0$ and $n=1$.

2. PRELIMINARY RESULTS.

Consider the equation

$$
\Delta_{n} Y(r, \theta)=\frac{\partial P}{\partial t}
$$

where $r^{2}=x_{1}^{2}+x_{2}^{2}+\cdots+x_{n}^{2}$ and $\theta=\tan ^{-1}\left(r / x_{n}\right)$. Then we have 


$$
\frac{\partial^{2} \omega}{\partial r^{2}}+\frac{n-1}{r} \frac{\partial r}{\partial r}+\frac{1}{r^{2} \sin ^{n-2} \theta} \frac{\partial}{\partial \theta}\left[\sin ^{n-2} \theta \frac{\partial r}{\partial \theta}\right]=\frac{\partial t}{\partial t} \text {. }
$$

Suppose the solution is of the type

$$
\boldsymbol{Y}(r, \theta)=u(r, t) p(\theta),
$$

then

$$
p(\theta)\left[\frac{\partial^{2} u}{\partial r^{2}}+\frac{n-1}{r} \frac{\partial u}{\partial r}+\frac{1}{r^{2} \sin ^{n-2} \theta} \frac{d}{d \theta}\left[\sin ^{n-2} \theta \frac{d p}{d \theta}\right] u\right]=\frac{\partial u}{\partial t} p(\theta)
$$

Letting

$$
\frac{1}{p(\theta) \sin ^{n-2} \theta} \frac{d}{d \theta}\left[\sin ^{n-2} \theta \frac{d p}{d \theta}\right]=-\alpha^{2},
$$

we finally have

$$
\frac{\partial^{2} u}{\partial r^{2}}+\frac{2 u}{r} \frac{\partial u}{\partial r}-\frac{\alpha^{2}}{r^{2}}=\frac{\partial u}{\partial t}
$$

where $n=2 \nu+1$, the generalized heat equation. Now from (2.1), we have

or

$$
\begin{aligned}
& \frac{1}{\sin ^{n-2} \theta} \frac{d}{d \theta}\left[\sin ^{n-2} \theta \frac{d p}{d \theta}\right]=-\alpha^{2} p(\theta), \\
& \frac{d^{2} p}{d \theta^{2}}+(n-2) \cot \theta \frac{d p}{d \theta}=-\alpha^{2} p .
\end{aligned}
$$

Let $\xi=\cos \theta$, then from above, we obtain

$$
\left(1-r^{2}\right) \frac{d^{2} p}{d \xi^{2}}-(n-1) \xi \frac{d p}{d \xi}=-\alpha^{2} p
$$

which has a solution

$$
p(\xi)=\left(\xi^{2}-1\right)^{-\frac{1}{2}} P_{\nu}^{m}(\xi),
$$

where $m=\frac{1}{2}(n-3), \quad \alpha^{2}=(\nu-m)(\nu+m+1)$ and $P_{\nu}^{m}(\xi)$ is the Legendre function of the first kind, [2,p.122]. Also by elementary methods [cf.6], we can find the solution of (2.2) as

$$
u(r, t)=\int_{0}^{\infty} U(s, r: t) u(s, 0) d s,
$$

where

$$
U(s, r: t)=\frac{1}{2 t} s^{\nu+\frac{1}{2}} r^{\frac{1}{2}-\nu} e^{-\frac{1}{4 t}\left(s^{2}+r^{2}\right)} I_{\mu}\left[\frac{s r}{2 t}\right],
$$

where $\mu^{2}=\left(\nu-\frac{1}{2}\right)^{2}+\alpha^{2}$, and $I_{\mu}(z)$, the usual modified Bessel function of the first kind. We shall call the functioon $U$ to be the source solution of the heat equation (2.2). If $U$ is considered as the kernel, then for a suitable $f$, its heat transform $F$ is defined by

$$
r^{k} F(r, t)=\int_{0}^{\infty} U(s, r: t) s^{k} f(s) d s,
$$

where $k=\mu+\frac{1}{2}-\nu$ and $F(r, 0)=f(r)$, the initial temperature. Numerous properties of the heat transform have been given in [6]. We note.that its inversion is given by

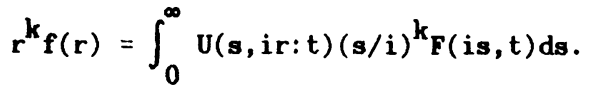

Suppose now that the initial temperature is the power function

$$
\begin{aligned}
& f(r)=r^{m} \text {, real and positive, then from (2.3), its heat transform, } \\
& \qquad \begin{aligned}
P_{m, \mu}(r, t) & =\int_{0}^{\infty} U(s, r,: t) s^{k+m} d s \\
& =\frac{\Gamma\left(\mu+\frac{1}{2}+1\right)}{\Gamma(\mu+1)}(4 t)^{\frac{1}{2}} r_{1}^{k} F_{1}\left[-\frac{1}{2} m ; \mu+1 ;-\frac{r^{2}}{4 t}\right]
\end{aligned}
\end{aligned}
$$


$\mu>-1, \quad t>0,\left[\begin{array}{ll}8 & \text { p.394}\end{array}\right]$. Thus giving a solution of $(2.2)$ involving the Hypergeometric function ${ }_{1} F_{1}$. As a special ease, if

$$
\text { m }=2 n, n=0,1,2, \ldots
$$

then

$$
P_{2 n, \mu}(r, t)=n !(4 t)^{n} r^{k} L_{n}^{\mu}\left(-r^{2} / 4 t\right)=(4 t)^{n} r^{k} \sum_{p=0}^{n} \frac{\Gamma(\mu+n+1)}{\Gamma(\mu+p+1)}\left[\begin{array}{l}
n \\
p
\end{array}\right]\left[\begin{array}{l}
x^{2} \\
4 t
\end{array}\right]^{p}
$$

defining the heat polynomial of degree $2 n$ in $r$ and of degree $n$ in $t$, involving the Laguerre polynomial. If we let $k=0$, we have the special case given in [4].

Next we define the Appell transfor of $P_{w, \mu}(r, t)$, real and positive as,

$$
\begin{aligned}
W_{m, \mu}(r, t) & =\operatorname{Ap}\left[P_{m, \mu}(r, t)\right] \\
& =H_{\mu}(0, r: t) P_{m, \mu}\left(\frac{r}{t},-\frac{1}{t}\right),
\end{aligned}
$$

where $H_{\mu}$, the Green's function, is defined by

and

$$
\begin{aligned}
& U(s, r: t)=s^{\mu+\nu+\frac{1}{2}}(r / t) k_{\mu}(s, r: t), \\
& H_{\mu}(s, r: t)=\frac{t^{k-1}}{2(s r)^{\mu}} e^{-\frac{s^{2}+r^{2}}{4 t}} I_{\mu}\left[\frac{s r}{2 t}\right] .
\end{aligned}
$$

It can be seen readily that

$$
w_{m, \mu}(r, t)=H_{\mu}(0, r: t) t^{-m-k} P_{m, \mu}(r,-t) .
$$

Now $H_{\mu}(0, r: t)=\frac{1}{2^{2 r+1} r(\mu+1)} t^{-v-\frac{1}{2}} e^{-r^{2} / 4 t}$,

therefore we can write

where $k=\mu+\nu-\frac{1}{2}$.

$$
w_{\mathbf{m}, \mu}(r, t)=\frac{1}{2^{2 \mu+1} \Gamma(\mu+1)} t^{-(m+\mu+1)} e^{-r^{2} / 4 t} P_{m, \mu}(r,-t),
$$

3. PROPERTIBS OF $P_{n, \mu}(r, t)$ AND $w_{n, \mu}(r, t)$.

In this section we shall establish various results involving the function $P_{2 n, \mu}(r, t)$ and its Appell transform $w_{2 n, \mu}(r, t)$. Using the asymptotic expansions, it is an easy matter to calculate the following estimates:

$$
\begin{aligned}
U(s, r: t)=0\left(|s|^{\nu} e^{-\frac{1}{4 t}(s-r)^{2}}\right. & \text { as }|s| \rightarrow \infty \\
P_{2 n, \mu}(r, t)=0\left(r^{2 n+k}\right) & \text { as } r \rightarrow \infty \\
P_{2 n, \mu}(r, t)=0\left[\frac{4 n t}{e}\right]^{n} & \text { as } n \rightarrow \infty .
\end{aligned}
$$

LEMMA 1. For $0 \leq x\langle\infty, t\rangle 0$,

$$
\int_{0}^{\infty} U(s, r: t) P_{2 n, \mu}(s,-t) d s=r^{k+2 n} \text {. }
$$

PROOF. Using the above estimates, note that the integral converges. Now, using (2.6) the definition of $P_{2 n, \mu}$ twice, we have,

$$
\begin{gathered}
\int_{0}^{\infty} U(s, r: t) P_{2 n, \mu}(s,-t) d s \\
=\sum_{p=0}^{n} \frac{\Gamma(\mu+n+1)}{\Gamma(\mu+p+1)}(-4 t)^{n-p}\left[\begin{array}{l}
n \\
p
\end{array}\right] \int_{0}^{\infty} U(s, r: t) s^{k+2 p} d s \\
=\sum_{p=0}^{n} \frac{\Gamma(\mu+n+1)}{\Gamma(\mu+p+1)}(-4 t)^{n-p}\left[\begin{array}{l}
n \\
p
\end{array}\right] P_{2 p, \mu}(r, t)
\end{gathered}
$$


Now consjder the inner su

$$
\begin{aligned}
& =\sum_{p=0}^{n} \frac{\Gamma(\mu+n+1)}{\Gamma(\mu+p+1)}(-4 t)^{n-p}\left[\begin{array}{l}
n \\
p
\end{array}\right]_{m=0}^{p} \frac{\Gamma(\mu+p+1)}{\Gamma(\mu+m+1)}(4 t)^{p-m}\left[\begin{array}{l}
p \\
m
\end{array}\right] r^{k+2 m} \\
& =\sum_{m=0}^{n}(-1)^{n} \frac{\Gamma(\mu+n+1)}{\Gamma(\mu+m+1)}(4 t)^{n-m} r_{p=m}^{k+2 m} \sum_{p=m}^{n}(-1)^{p}\left[\begin{array}{l}
n \\
p
\end{array}\right]\left[\begin{array}{l}
p \\
m
\end{array}\right]
\end{aligned}
$$

$$
\begin{gathered}
\sum_{p=m}^{n}(-1)^{p}\left[\begin{array}{l}
n \\
p
\end{array}\right]\left[\begin{array}{l}
p \\
w
\end{array}\right]=\sum_{i=0}^{n-m}(-1)^{i+m}\left[\begin{array}{c}
n \\
i+n
\end{array}\right]\left[\begin{array}{c}
i+m \\
m
\end{array}\right] \\
=(-1)^{m} \frac{n !}{m !} \sum_{i=0}^{n-m} \frac{(-1)^{i}}{i !(n-m-i) !}=\frac{(-1)^{n} n !}{m ! l !} \sum_{i=0}^{l}(-1)^{i}\left[\begin{array}{l}
l \\
i
\end{array}\right] .
\end{gathered}
$$

Thus the inner sum is 0 if $\ell \neq 0$ and $l$ if $\ell=0$ i.e. if $m=n$. Thearefore (3.2), reduces to $r^{k+2 n}$ and hence

$$
\int_{0}^{\infty} U(s, r: t) P_{2 n, \mu}(s,-t) d s=r^{k+2 m}
$$

as desired.

The equation (3.1) gives us an inversion formula of (2.5) with $m=2 n$. We now derive a generating function for $P_{2 n, \mu}(r, t)$

LEMMA 2. For $0 \leq x<\infty,-\infty<t<\infty, y<\frac{1}{4 t}$,

$$
\sum_{n=0}^{\infty} \frac{y^{n}}{n !} P_{2 n, \mu}(r, t)=\frac{r^{k}}{(1-4 y t)^{\mu+1}} e^{\frac{r^{2} y}{1-4 y t}}, k=\mu-\nu+\frac{1}{2} .
$$

ProOF. Let $t>0$. Using (2.5) and (2.3), we have

$$
\begin{aligned}
\sum_{n=0}^{\infty} \frac{y^{n}}{n !} P_{2 n, \mu}(r, t) & =\sum_{n=0}^{\infty} \frac{y^{n}}{n !} \int_{0}^{\infty} U(s, r: t) s^{k+2 n} d s \\
& =\int_{0}^{\infty} U(s, r: t) s^{k} \sum_{n=0}^{\infty} \frac{\left(s^{2} y\right)^{n}}{n !} d s \\
& =\int_{0}^{\infty} U(s, r: t) s^{k} e^{s^{2} y} d s \\
& =\frac{1}{2 t} r^{\frac{1}{2}} e^{-r^{2} / 4 t} \int_{0}^{\infty} s^{k+\nu+\frac{1}{2}} e^{-s^{2}\left(\frac{1}{4 t}-y\right)} I_{\mu}\left[\frac{s r}{2 t}\right] d s \\
& =\frac{r^{k}}{(1-4 t)^{\mu+1}} e^{\frac{r^{2} y}{1-4 y t},}
\end{aligned}
$$

[8,p.394] as required. The interchange of sumation and integration is valid since

$$
\int_{0}^{\infty} s^{k+\nu+\frac{1}{2}} e^{-s^{2}\left(\frac{1}{4 t}-y\right)} I_{\mu}\left[\frac{s r}{2 t}\right] d s<\int_{0}^{\infty} s^{k+\nu+\frac{1}{2}} e^{-s^{2}\left(\frac{1}{4 t}-y\right)} \cdot e^{\frac{s r}{2 t}} d s<\infty .
$$

If $t=0$, the result can easily be computed, since $P_{2 n, \mu}(r, 0)=r^{k+2 n}$. For $t<0$, we use the fact that

$$
P_{2 n, \mu}(r,-t)=i^{2 n-k} P_{2 n, \mu}(i r, t)
$$

from its representation given in (2.6). The lemma is then proved on the same lines as for the case $t>0$.

Now we give a generating function for $w_{2 n, \mu}(r, t)$, the Appell transform of $\mathbf{P}_{2 n, \mu}(r, t)$.

LEMMA 3. For $t \geq 0,|z|<\frac{1}{4} t$, and $k=\mu-\nu+\frac{1}{2}$,

$$
\sum_{n=0}^{\infty} \frac{z^{n}}{n !} w_{2 n, \mu}(r, t)=\left[\frac{r}{t+4 z}\right]^{k} H_{\mu}(0, r: t+4 z),
$$


PROOF. Note that $w_{2 n, \mu}(r, t)=0\left[\frac{4 n}{e t}\right]^{n}$, as $n \rightarrow \infty$, and hence the series converges absolutely when $|z|<\frac{1}{4} t$. Using (2.8), we have

$$
\begin{aligned}
\sum_{n=0}^{\infty} \frac{z^{n}}{n !} W_{2 n, \mu}(r, t) & =H_{\mu}(0, r: t) t^{-k} \sum_{n=0}^{\infty} \frac{1}{n !}\left(z / t^{2}\right)^{n} P_{2 n, \mu}(r,-t) \\
& =H_{\mu}(0, r: t) t^{-k} k\left[\frac{t}{t+4 z}\right]^{\mu+1} e^{\frac{r^{2} z}{t(t+4 z)}}, \\
& =\left[\frac{r}{t+4 z}\right]^{k} H_{\mu}(0, r: t+4 z),
\end{aligned}
$$

due to Lemma 2 and making use of the definition of $H_{\mu}$ given by (2.9).

If we expand the right hand side of (3.4) by Taylor series in powers of $z$, we have

$$
\left[\frac{r}{t+4 z}\right]^{k} H_{\mu}(0, r: t+4 z)=\sum_{n=0}^{\infty} \frac{(4 z)^{n}}{n !}\left[\frac{\partial}{\partial t}\right]^{n}\left[\left[\frac{r}{t}\right]^{k} H_{\mu}(0, r: t)\right]
$$

On comparing this series with the series on the left hand side of (3.4), we obtain

$$
\begin{aligned}
& w_{2 n, \mu}(r, t)=2^{2 n}\left[\frac{\partial}{\partial t}\right]^{n}\left[\left[\frac{r}{t}\right]^{k} H_{\mu}(0, r: t)\right] \\
& =2^{2 n}\left[\frac{\partial}{\partial t}\right]^{n}\left[\left[\frac{r}{t}\right]^{k} \cdot \frac{t^{-\nu-\frac{1}{2}}}{2^{2 \mu+1} r(\mu+1)} e^{-r^{2} / 4 t}\right] \text {, } \\
& =\frac{2^{2 n-\mu}}{\Gamma(\mu+1)}\left[\frac{\partial}{\partial t}\right]^{n} r^{k-\mu} \int_{0}^{\infty} J_{\mu}(r u) e^{-t u^{2}} u^{\mu+1} d u \\
& =\frac{2^{2 n-\mu}}{\Gamma(\mu+1)} \mathbf{r}^{k-\mu} \int_{0}^{\infty} J_{\mu}(r u) u^{\mu+1}\left[\frac{\partial}{\partial t}\right]^{t}\left[e^{-\operatorname{tn}^{2}}\right] d u \\
& =\frac{(-1)^{n} 2^{2 n-\mu}}{\Gamma(\mu+1)} \mathbf{r}^{\frac{1}{2} \nu} \int_{0}^{\infty} J_{\mu}(r u) u^{2 n+\mu+1} e^{-t u^{2}} d u \text {, }
\end{aligned}
$$

giving us an integral representation for $w_{2 n, \mu}(r, t)$.

Also we give other generating functions for the function $P_{2 n, \mu}(r, t)$ and its Appell transform $W_{2 n, \mu}(r, t)$. We shall simply write down the results, which can be proved following a similar analysis as used for the Lemas 2 and 3 above.

LEMMA 4. For $\rightarrow<t<\infty$ and all complex $z$,

$$
\sum_{n=0}^{\infty} \frac{z^{2 n}}{n ! \Gamma(\mu+n+1)} P_{2 n, \mu}(r, t)=z^{-\mu} r^{\frac{1}{2}-\nu} e^{4 t z^{2}} I_{\mu}(2 x z) \text {. }
$$

LFMMA 5. For $-\infty<t<\infty$ and all complex $z$,

$$
\sum_{n=0}^{\infty} \frac{\Gamma(\mu+1)}{n ! \Gamma(\mu+n+1)}\left[\frac{z}{4}\right]^{2 n} W_{2 n, \mu}(r, t)=\left[\frac{r}{t}\right]^{k} H_{\mu}(z, r: t) .
$$

Now we shall prove an important property of the sets of functions $P_{2 n, \mu}(r, t)$ and $W_{2 n, \mu}(r, t)$ and show that they form a biorthogonal system.

THEOREM. For $t>0$,

$$
\int_{0}^{\infty} P_{2 m, \mu}(x,-t) W_{2 n, \mu}(x,-t) x^{2 \nu} d x=\frac{\Gamma(\mu+n+1)}{\Gamma(\mu+1)} n ! 4^{2 n} \delta_{m},
$$

where $\delta_{m n}$ is the Dirac-delta function.

PROOF. Using (2.8),

$$
\int_{0}^{\infty} P_{2 n, \mu}(x,-t) w_{2 n, \mu}(x,-t) x^{2 \nu} d x
$$


due to (2.6).

$$
\begin{gathered}
=\int_{0}^{\infty} H_{\mu}(0, x: t) t^{-2 n-\mu} P_{2 n, \mu}(x,-t) P_{2 m, \mu}(x,-t) x^{2 \nu} d x \\
=\frac{1}{2^{2 \mu+1} \Gamma(\mu+1)} t^{m-n-\mu-1} n ! m !(-4)^{m+n} \int_{0}^{\infty} x^{2 \mu+1} e^{-x^{2} / 4 t} L_{n}^{\mu}\left[\frac{x^{2}}{4 t}\right] L_{m}^{\mu}\left[\frac{x^{2}}{4 t}\right] d x,
\end{gathered}
$$

The integral on the right handside of (3.6) with a change of variable can be written as, $[3, p .188]$.

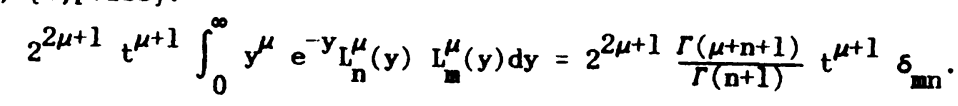

Hence the right hand side of (3.8) gives,

$$
\begin{aligned}
& \frac{\Gamma(\mu+n+1)}{\Gamma(\mu+1)} \mathrm{t}^{m-n} m !(-4)^{m+n} \delta_{m n} \\
= & \frac{\Gamma(\mu+n+1)}{\Gamma(\mu+1)} n ! 4^{2 n} \delta_{m n},
\end{aligned}
$$

as required.

Next we shall establish a generating function for the biorthogonal set $P_{2 m, \mu}(x, t) w_{2 n, \mu}(x, t)$.

LBMMA 6. For $x, y, s$ and $t>0$ and $\left|z^{2} t\right|<s$,

$$
\sum_{n=0}^{\infty} \frac{r(\mu+1)}{n ! r(\mu+n+1)}\left[\frac{z}{4}\right]^{2 n} P_{2 n, \mu}(x, t) w_{2 n, \mu}(y, s) /=\left[\frac{x y}{s+z^{2}{ }_{t}}\right]^{k} H_{\mu}\left(x z, y: s+z^{2} t\right) .
$$

PROOF. Note that the series converges for $\left|z^{2} t\right|<s$, using the asymptotic estimates of the functions $P_{2 n, \mu}$ and $W_{2 n, \mu}$ therefore,

$$
\begin{aligned}
& \sum_{n=0}^{\infty} \frac{\Gamma(\mu+1)}{n !(\Gamma(\mu+n+1)}\left[\frac{z}{4}\right]^{2 n} P_{2 n, \mu}(x, t) W_{2 n, \mu}(y, s) \\
& =\sum_{n=0}^{\infty} \frac{(-1)^{n} y^{\frac{1}{2}-\nu}}{2^{n} n ! r(\mu+n+1)} P_{2 n, \mu}(x, t) \int_{0}^{\infty}(2 \mu)^{\mu+2 n+1} e^{-s u^{2} J_{\mu(y u) d u,}}
\end{aligned}
$$

due to (3.5),

$$
\begin{aligned}
& =2 y^{\frac{1}{2}-\nu} \int_{0}^{\infty} u^{\mu+1} e^{-s u^{2}} J_{\mu}(y u) d u \sum_{n=0}^{\infty} \frac{1}{n ! \Gamma(\mu+n+1)}\left[\frac{u z i}{2}\right]^{2 n} P_{2 n, \mu}(x, t) \\
& =2^{\mu+1} z^{-\mu}(x y)^{\frac{1}{2}-\nu} \int_{0}^{\infty} u e^{-u^{2}\left(s+z^{2} t\right)} J_{\mu}(x u z) J_{\mu}(y u) d u \\
& =\frac{2^{\mu}}{s+z^{2} t}(x y)^{\frac{1}{2}-\nu} z^{-\mu} e^{\frac{y^{2}+x^{2} z^{2}}{4\left(s+z^{2} t\right)}} I_{\mu}\left[\frac{x y z}{2\left(s+z^{2} t\right)}\right],[1, p \cdot 51] . \\
& =\left[\frac{x y}{s+z^{2} t}\right]^{k} H_{\mu}\left(x z, y: s+z^{2} t\right),
\end{aligned}
$$

due to the definition in (2.7).

Now two results on finite sums involving the functions $P_{2 n, \mu}$ and $w_{2 n, \mu}$.

LBMMA 7. For $t>0, \mu>0$, and a complex $z$,

$$
\sum_{m=0}^{n} \frac{(-1)^{m}}{m !}\left[\begin{array}{c}
n+\mu \\
n-m
\end{array}\right] z^{m} P_{2 n, \mu}(r, t)=r^{k}(1-4 t z)^{n_{2}} L_{n}^{\mu}\left[\frac{z r^{2}}{1-4 z t}\right] \text {. }
$$

PROOF. By (2.5), $\sum_{m=0}^{n} \frac{(-1)^{m}}{! !}\left[\begin{array}{l}n+\mu \\ n-m\end{array}\right] z^{m} P_{2 n, \mu}(r, t)$

$$
\begin{aligned}
& =\sum_{m=0}^{n} \frac{(-1)^{m}}{m !}\left[\begin{array}{l}
n+\mu \\
n-m
\end{array}\right] z^{m} \int_{0}^{\infty} U(s, r: t) s^{k+2 m} d s \\
& =\int_{0}^{\infty} U(s, r: t) s^{k} d s \cdot \sum_{m=0}^{n} \frac{(-1)^{m}}{m !}\left[\begin{array}{l}
n-\mu \\
n-m
\end{array}\right]\left(z^{2}\right)^{m}
\end{aligned}
$$




$$
\begin{aligned}
& =\int_{0}^{\infty} U(s, r: t) s^{k} L_{n}^{\mu}\left(z s^{2}\right) d s, \\
& =\frac{1}{2 t} r^{\frac{1}{2}-\nu} e^{\frac{-r^{2}}{4 t}} \int_{0}^{\infty} s^{\mu+1} e^{-s^{2} / 4 t} I_{\mu}\left[\frac{s r}{2 t}\right] L_{n}^{\mu}\left(z s^{2}\right) d s \\
& =r^{k}(1-4 t z)^{n} L_{n}^{\mu}\left[\frac{z r^{2}}{1-4 z t}\right],[1, p .43],
\end{aligned}
$$

as required.

A similar result can also be proved involving $w_{2 n, \mu}$.

LEMMA 8. For $t>0, \mu>0$ and a complex $z$,

$$
\sum_{m=0}^{n} \frac{(-1)^{m}}{m !}\left[\begin{array}{c}
n+\mu \\
n-m
\end{array}\right] z^{m} w_{2 m, \mu}(r, t)=\frac{r^{k}}{2^{\mu+1} \Gamma(\mu+1)} \cdot t^{-(n+\mu+1)} e^{-r^{2} / 4 t}(t+4 z)^{n} L \frac{z r^{2}}{n}\left[\frac{t+4 z)}{t(t)}\right.
$$

\section{SERIES RBPRESBNTATION}

In this section we shall establish a series representation of the heat transform $F(r, t)$ in terms of Laguerre polynomials and confluent hypergeometric functions.

As mentioned earlier, for a suitable $f$, its heat transform $F$ is given by

$$
r^{k} F(r, t)=\int_{0}^{\infty} U(s, r: t) s^{k} f(s) d s, t>0,
$$

where $F(r, 0)=f(r)$ and $r^{k} F(r, t)$ is a solution of the generalized heat equation

THBAOREM: If $f(x)=\sum_{n=0}^{\infty} a_{n} x^{n}$, has a growth $\left[1, \frac{e}{4 \sigma}\right], \sigma>0$, then

$$
r^{k} F(r, t)= \begin{cases}\int_{0}^{\infty} U(s, i r:-t)(s / i)^{k} f(i s) d s, & -0<t<0 \\ \int_{0}^{\infty} U(s, r: t) s^{k} f(s) d s, & 0<t<0\end{cases}
$$

where $k=\mu-\nu+\frac{1}{2}$.

PROOF. If $0<t<6$, we have

$$
\begin{aligned}
r^{k} F(s, t) & =\int_{0}^{\infty} U(s, r: t) s^{k} \sum_{n=0}^{\infty} a_{n} s^{n} d s \\
& =\sum_{n=0}^{\infty} a_{n} \int_{0}^{\infty} U(s, r: t) s^{k+n} d s \\
& =\sum_{n=0}^{\infty} a_{n} P_{n, \mu}(r, t),
\end{aligned}
$$

due to (2.5). The interchange of sumation and integration is valid since

$$
\int_{0}^{\infty}\left|U(s, r: t) s^{k+n}\right| d s<\int_{0}^{\infty} e^{-\frac{1}{4 t}(s+r)^{2}} s^{k+\nu+n+1 / 2} d s<\infty .
$$

Also, if $-\delta<t<0$,

due to (3.3). Hence the result

$$
\begin{aligned}
\int_{0}^{\infty} U(s, i r:-t) & (s / i)^{k} f(s) d s=\int_{0}^{\infty} U(s, i r:-t)(s / i)^{k} \sum_{n=0}^{\infty} a_{n}(i s)^{n} d s \\
& =\sum_{n=0}^{\infty} a_{n} i^{n-k} \int_{0}^{\infty} U(s, i r:-t) s^{k+n} d s \\
& =\sum_{n=0}^{\infty} a_{n} i^{n-k} P_{n, \mu}(i r,-t)=\sum_{n=0}^{\infty} a_{n} P_{n, \mu}(r, t),
\end{aligned}
$$

Furthermore, for $0<|t|<\delta$, 


$$
r^{k} F(r, t)=\sum_{n=0}^{\infty} a_{n} P_{n, \mu}(r, t)
$$

or,

$$
r^{k} F(r, t)=\sum_{n=0}^{\infty} a_{2 n} P_{2 n, \mu}(r, t)+\sum_{n=0}^{\infty} a_{2 n+1} P_{2 n+1, \mu}(r, t) .
$$

Now making use of the definitions given in (2.5) and (2.6), we obtain

$$
F(r, t)=\sum_{n=0}^{\infty} a_{2 n} n !(4 t)^{n} L_{n}^{\mu}\left(-r^{2} / 4 t\right)+\sum_{n=0}^{\infty} a_{2 n+1} \frac{\Gamma\left(\mu+n+\frac{3}{2}\right)}{\Gamma(\mu+1)}(4 t)^{n+\frac{1}{2}} \cdot F_{1}\left[-n-\frac{1}{2}: r+1:-\frac{r^{t}}{4 t}\right],
$$

giving us a representation involving Laguerre polynomial and confluent hypergeometric function.

If we set $a=0$ i.e. $\mu=\nu \cdot-\frac{1}{2}$ and $k=0$, throughout, most of the results derived here, reduce to known results given in [4] and [5]. Further, if we set $\nu=0$, i.e. $n=1$, the results coincide with those derived in [7].

ACKNOWLEDGBMENT. This research is partially supported by a grant from Natural Sciences and Bngineering Research Council of Canada.

\section{REFBRBNCES}

1. A Erdelyi et al. "Tables of Integral Transforms, Vol. 2", Mc Graw Hill, Toronto, 1954 .

2. A. Erdelyi et al. "Higher Transcendental Functions, Vol. 1", McGraw Hill, Toronto, 1954 .

3. A Brdelyi et al. "Higher Transcendental Functions, Vol. 2", McGraw Hill, Toronto, 1954 .

4. D.T. Haimo, "Expansions in terms of generalized heat polynomials and of their Appell transfore", J. Math. \& Mech. Vol. 15, No. 5, (1966), 735-758.

5. D.T. Haimo, "Series expansion and integral representation of generalized temperataures", Illinois J. Math. Vol. 14, No. 4, (1970), 621-629.

6. C. Nasim and B.D. Aggarwala, "On the generalized heat equation", Proc. Bdinburgh Math. Soc. (1984), 27, 261-273.

7. P.C. Rosenbloom and D.V. Widder, "Expansions in tearms of heat polynomials and associated functions", Trans. Amer. Math. Soc., Vol. 92 (1959), 220-266.

8. G.N. Watson, "Theory of Bessel functions", Second Edition, University Press, Cambridge, 1966. 


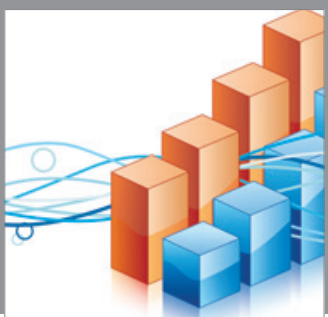

Advances in

Operations Research

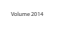

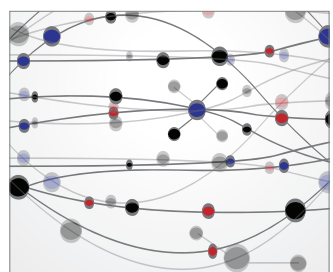

\section{The Scientific} World Journal
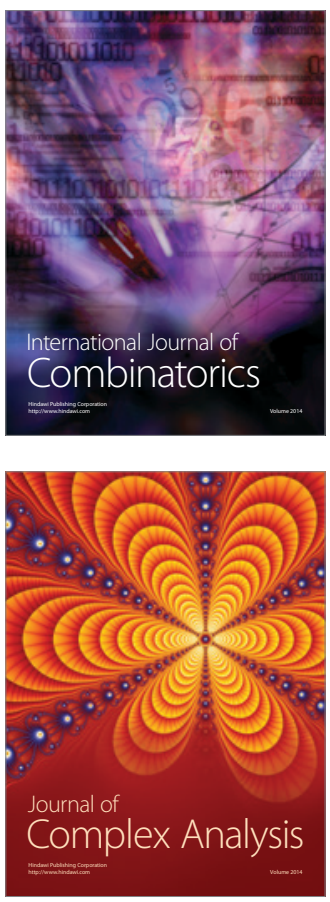

International Journal of

Mathematics and

Mathematical

Sciences
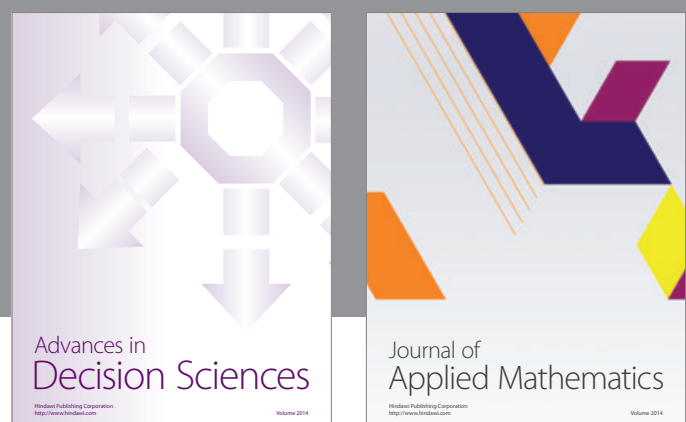

Journal of

Applied Mathematics
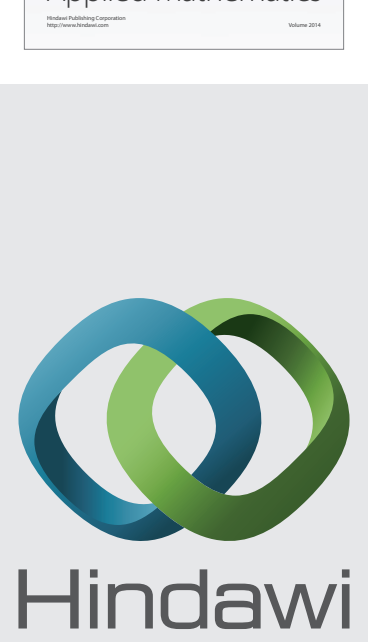

Submit your manuscripts at http://www.hindawi.com
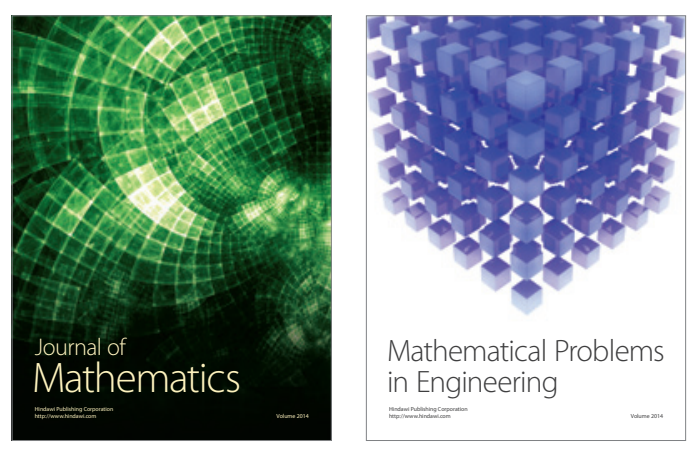

Mathematical Problems in Engineering
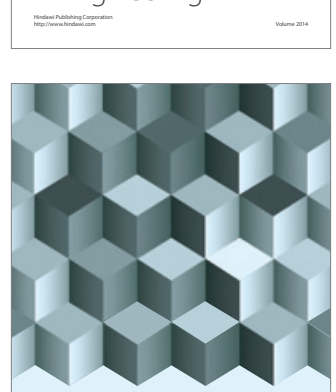

Journal of

Function Spaces
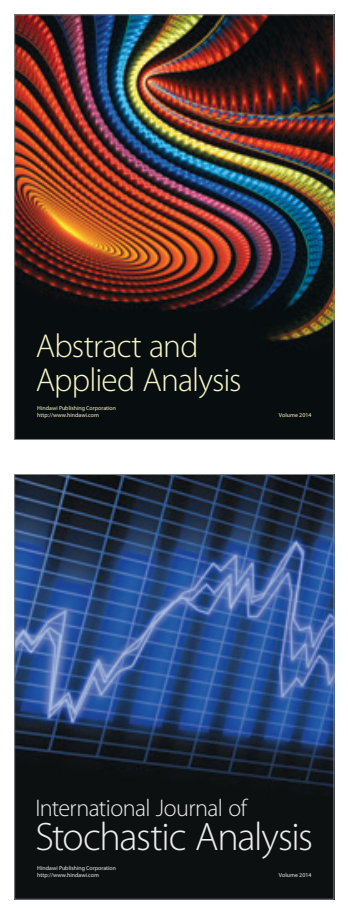

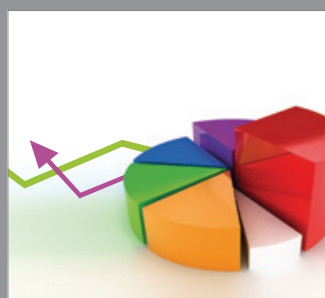

ournal of

Probability and Statistics

Promensencen
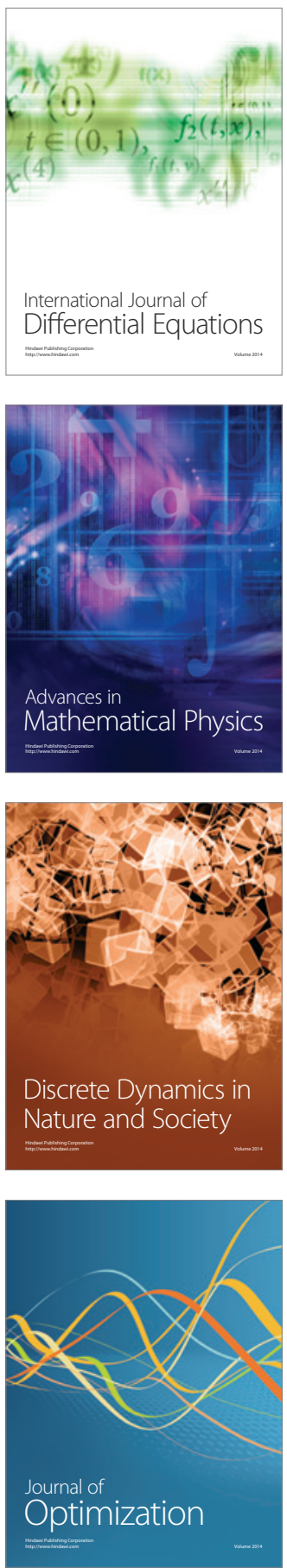